\title{
Bacteriophage therapy for infections in $\mathrm{CF}$
}

\author{
Benjamin Chan ${ }^{1}$, Gail Stanley ${ }^{2}$, Mrinalini Modak ${ }^{3}$, Jon Koff ${ }^{2}$, and Paul Turner ${ }^{1}$ \\ ${ }^{1}$ Yale University \\ ${ }^{2}$ Yale School of Medicine \\ ${ }^{3}$ Yale-New Haven Hospital
}

August 7, 2020

\begin{abstract}
Abstract. Pseudomonas aeruginosa and Staphylococcus aureus are bacterial pathogens frequently associated with pulmonary complications and disease progression in cystic fibrosis (CF) patients. However, these bacteria increasingly show multiple resistance to antibiotics, necessitating novel management strategies. One possibility is phage therapy, where lytic bacteriophages (phages; bacteria-specific viruses) are administered to kill target bacterial pathogens. Recent publication of case reports of phage-therapy treatment of antibiotic-resistant lung infections in CF has garnered significant attention. These cases exemplify the renewed interest in phage therapy, as an older concept that is newly updated to include rigorous collection and analysis of patient data to assess clinical benefit, while informing the development of clinical trials. As outcomes of these trials become public, the results will valuably gauge the potential usefulness of phage therapy to address the rise in antibiotic-resistant bacterial infections. In addition, we highlight the further need for basic research on accurately predicting the different responses of target bacterial pathogens when phages are administered alone, sequentially or as mixtures (cocktails), and whether within-cocktail interactions among phages hold consequences for the efficacy of phage therapy in patient treatment.
\end{abstract}

\section{Bacteriophage therapy for infections in CF}

Benjamin K. Chan ${ }^{1}$, Gail Stanley ${ }^{2}$, Mrinalini Modak ${ }^{3}$, Jon L. Koff ${ }^{2}$, Paul E. Turner ${ }^{1,4, \#}$

${ }^{1}$ Department of Ecology and Evolutionary Biology, Yale University, New Haven, CT 06511

${ }^{2}$ Department of Internal Medicine, Yale School of Medicine, New Haven, CT 06520

${ }^{3}$ Yale New Haven Hospital, New Haven, CT 06520

4 Program in Microbiology, Yale School of Medicine, New Haven, CT 06520\# Correspondence, paul.turner@yale.edu

Abstract. Pseudomonas aeruginosa and Staphylococcus aureusare bacterial pathogens frequently associated with pulmonary complications and disease progression in cystic fibrosis (CF) patients. However, these bacteria increasingly show multiple resistance to antibiotics, necessitating novel management strategies. One possibility is phage therapy, where lytic bacteriophages (phages; bacteria-specific viruses) are administered to kill target bacterial pathogens. Recent publication of case reports of phage-therapy treatment of antibioticresistant lung infections in CF has garnered significant attention. These cases exemplify the renewed interest in phage therapy, as an older concept that is newly updated to include rigorous collection and analysis of patient data to assess clinical benefit, while informing the development of clinical trials. As outcomes of these trials become public, the results will valuably gauge the potential usefulness of phage therapy to address the rise in antibiotic-resistant bacterial infections. In addition, we highlight the further need for basic research on accurately predicting the different responses of target bacterial pathogens when phages are administered 
alone, sequentially or as mixtures (cocktails), and whether within-cocktail interactions among phages hold consequences for the efficacy of phage therapy in patient treatment.

Extensive antibiotic use during the previous century has resulted in an alarming rise in antibiotic resistant infections. These infections, particularly for bacterial pathogens which are resistant to multiple classes of antibiotics [also known as multi-drug resistant (MDR)], significantly contribute to increased morbidity and mortality [1]-[4]. As a result, we have been forced to increase our reliance on drugs of last resort [5], [6]. Predictably, bacteria have now emerged that are resistant to even these drugs [5], [6]. While in some cases these isolates may not have spread widely, concern is warranted, and the development of new antibiotics and management strategies is urgently needed.

While antibiotic resistance increases, the antibiotic development pipeline is lagging far behind with limited evidence that novel antibiotics are being discovered and developed [7]. Therefore, efforts by the World Health Organization have resulted in the creation of a list of 'priority pathogens' for antibiotic development, due to their public health relevance. This list contains six bacterial pathogens notable for their high levels of antibiotic resistance, as well as their ability to escape conventional therapies. These bacteria are Enterococcus faecium, Staphylococcus aureus, Klebsiella pneumoniae, Acinetobacter baumanii ,Pseudomonas aeruginosa , and Enterobacter spp. are named by the ESKAPE acronym because they are becoming increasingly prevalent as MDR and pan-drug resistant (PDR) organisms that frequently escape approved treatments. For cystic fibrosis $(\mathrm{CF})$ patients and their care teams, $P$. aeruginosa and $S$. aureus are common pathogens associated with pulmonary complications and disease progression. Because of the need to frequently treat pulmonary exacerbations with antibiotics the $\mathrm{CF}$ community has been wrestling with the challenges of increased antibiotic resistance for some time.

S. aureus is a Gram-positive bacterium that is highly relevant in the CF lung, where it is frequently the first bacterial pathogen cultured from those with CF [8]. Strains with resistance to methicillin (i.e., methicillin resistant $S$. aureus [MRSA]), have been shown to correlate with more rapid pulmonary decline, relative to S. aureus strains which are sensitive to methicillin [9]. Attempts to actively eradicate MRSA upon detection have been successful, resulting in improved $\mathrm{FEV}_{1}$ and $\mathrm{BMI}$; however, spontaneous eradication in the absence of intervention was also observed in the same study [10]. Ultimately, presence of $S$. aureus can increase the risk of subsequent $P$. aeruginosa infection by damaging lung tissue and creating an environment favorable for growth [11]. Thus, because $S$. aureus is one of the first identified pathogens in the lungs of those with $\mathrm{CF}$ that paves the way for eventual $P$. aeruginosa infection, control strategies are needed.

$P$. aeruginosa is a Gram-negative bacterium that thrives in myriad natural and artificial environments, which vary from household sink drains to the natural environment (soil and water sources) to clinics and hospitals [12]. Thus, individuals with CF readily encounter $P$. aeruginosa in their home environment and when they receive medical care. While $P$. aeruginosa is less common in pediatric CF sputum, by adulthood, the majority of $\mathrm{CF}$ patients have $P$. aeruginosa in sputum, which negatively correlates with $\mathrm{CF}$ lung function [13], [14]. P. aeruginosa employs multiple strategies to colonize the CF lung, and several of these are virulence factors that induce lung inflammation and tissue damage, which may contribute to the severity of pulmonary exacerbations. While the CF community also suffers from a lack of new antibiotics, the recent development of CFTR modulators may affect sputum colonization of $P$. aeruginosa and the frequency of pulmonary exacerbations [15]. Although the long-term durability of these effects remains may be limited $[16]$.

While CF clinical outcomes and survival in the past 30 years has improved, the persistence of $S$. aureus, $P$. aeruginosa, and other Gram-negative bacteria (e.g., Burkholderia and Achromobacter ) that are increasingly resistant to antibiotics, and the emergence of non-tuberculous mycobacteria, highlight the need to develop novel antimicrobial therapeutics that, ideally, can also decrease the inflammation and tissue-damaging virulence factors. Ideally, such therapeutics will also have limited, if any, off-target effects, which would provide an intervention that greatly benefits the CF patients. One approach which has recently received an abundance of attention, particularly in the CF community, is phage therapy. 
Phage therapy is the use of lytic bacteriophages (viruses of bacteria) to kill infectious bacteria. Such an approach harnesses phages, which are abundant in nature, to efficiently and effectively kill specific bacteria. Discovered in the early 1900s, phages have been used for human therapeutics since then, but after the discovery of penicillin, phage therapy continued in the former Soviet Union and Eastern Europe more than Western countries. While much of the available data from this experience have been translated from Russian, these reports estimate that over 300,000 individuals have received phage therapy over the last 100 years. While there have been few reports of adverse events, access to the data is limited. Furthermore, general consensus for phage therapy is that it is safe in humans without evidence for toxicity [17]-[20].

Because of the emergence of MDR bacteria, phage therapy is again being seriously considered in Europe, the United States and elsewhere as a potential approach to manage antibiotic resistant infections. In addition to more clinical experience with phage therapy, which is summarized below, the CF Foundation has provided support to the Center for Innovative Phage Applications and Therapeutics at the University of California, San Diego, and funding for phage therapy clinical trials in CF from Armata Pharmaceuticals and Yale University (personal communication, J.P. Clancy M.D., Vice President of Clinical Research, CF Foundation).

The existing phage therapy clinical trials have studied infectious diarrhea, wound infections, and chronic otitis media with different metrics of efficacy, but no significant side effects. While no clinical trials have been completed in $\mathrm{CF}$ at this time, there are published reports of individuals with $\mathrm{CF}$ being treated with phage therapy before or after lung transplantation. In 2008, Kutateladze and Adamia described the treatment of pulmonary infections at the Eliava Institute of Bacteriophages, Microbiology, and Virology in Tbilisi, Georgia [21]. This included adding phages to CF care. CF patients included both infants and adults, who received phage therapy to treat $S$. aureusand $P$. aeruginosa by nebulizer over approximately 1 week. The authors reported that phage therapy caused decreased densities of sputum bacteria, improvements in patient health, and extension of time until subsequent bacterial infection. Kvachadze et al., reported a case of phage therapy in a pediatric CF patient who received phage therapy to treat $S$. aureus and $P$. aeruginosa via nebulization a total of 9 times once every 4 to 6 weeks [22]. Phage therapy was reported to result in reduction in bacterial titers and a $50 \%$ reduction in administered antibiotics over 9 months of treatment. While there was no change in chest imaging, both $S$. aureus and P. aeruginosawere undetectable after phage therapy. Subsequently, Aslam and colleagues reported their experience with phage therapy in three post-lung transplant patients [23]. One of these patients had CF whose post-transplant course was complicated with Burkholderia dolosa. Phage therapy was administered with antibiotics. Initial phage therapy resulted in decreased fever and leukocytosis with evidence for improved consolidations on chest imaging. Continued therapy over approximately six weeks led to improved functional status. However, $B$. dolosa after ten weeks resulted in pneumonia, sepsis, and ultimately multi-organ failure, and this patient was transitioned to hospice. A 26-year-old woman with CF listed for lung transplant was treated with intravenous phage cocktail of four lytic phages every six hours for eight weeks in addition to antibiotics for MDR P. aeruginosa. Phage therapy resulted in decreased supplemental oxygen use and reduced sputum production without evidence for recurrent exacerbation for 100 days, and this patient subsequently received lung transplant nine months later. Most recently, Dedrick et. al. [24], reported the use of phage cocktail, which included an engineered phage, to treat disseminated Mycobacterium abscessus in a fifteen year-old individual with $\mathrm{CF}$ who was post-lung transplant. In this case, phage therapy resulted in decreased size of skin lesions, and improvement in lung function, liver function, chest imaging and weight gain. Here at Yale, we've successfully treated multiple CF associated infections, observing a rapid decrease in sputum bacteria density and correlating with significant improvement of FEV1\%pred. This experience, and those of others have been invaluable as we and others proceed with clinical trials.

In summary, while the existing published literature in CF is limited, there are encouraging signs that phage therapy may provide clinical benefit, which needs to be confirmed in rigorous, controlled clinical trials. In addition, these cases highlight potential differences in route of phage administration (e.g., intravenous vs. nebulized), concomitant use of antibiotics, short-term or longer-term duration of therapy, choice of phage, and single phage vs. multi-phage "cocktails". These differences could have a major impact on outcomes, and through studies propelled by recent interest, the specific contribution of each approach can be identified. 
Above, we discuss how the resurged interest in phage therapy and promising outcomes from administered treatment in human patients highlight the need for abundant basic and applied research. Here, we highlight two examples that pertain to (i) effects of differing strategies for administering phages and whether these would cause problems in accurately predicting how target bacteria will evolutionarily respond, and (ii) cocktail approaches to phage therapy and how the supposed benefits of cocktails for covering 'genotype space' of target bacterial strains might be offset by the costs of phage-phage interactions that diminish cocktail efficacy.

Recently, Wright et al. [25] examined evolution of bacterial resistance to phages, by studying interactions between $P$. aeruginosa strain PA01 and phages that bind to host cells by utilizing either lipopolysaccharide (LPS) or the type-IV pilus. In particular, they assessed whether sequential exposure to the two different phages caused mutational evolution of phage resistance in the target bacterial population that was distinct from resistance evolution when the two phages were placed together in a cocktail (mixture). This possible differing outcome relates to the general idea that biological evolution in response to selection by a single environmental challenge (each phage alone), may differ from molecular evolution in response to the same environmental challenges when experienced simultaneously (phage mixture) (e.g., see Kassen 2002 on evolution in simple versus complex environments [26]) Results showed that the genetic basis of bacterial resistance in response to sequential phage exposure generally led to mutations in genes associated with the binding target (LPS or type-IV pilus), indicating evolution of additive resistance in the target bacterial populations. Whereas, this accumulation of multiple receptor-specific resistance mutations was never observed when phage pairs targeting different binding sites were applied simultaneously. Rather, half of the observed phage-cocktail-resistant mutants showed only LPS changes and no detected mutations in genes for the type-IV pilus; the remaining mutants either surprisingly presented no mutational changes or showed duplications in genes for bacterial recombination that could not easily explain their supposed relationship to evolved phage resistance. In this study, the data indicated that bacterial evolution in response to sequential phage exposure was both more predictable and interpretable, relative to bacterial evolution against the same phages when mixed together as a cocktail [25]. In turn, because phage-steering approaches are designed to leverage phage killing of target bacteria alongside predictable clinically-useful evolution of resistance tradeoffs in bacterial pathogens, the study by Wright et al. [25] suggests that administering phage cocktails can cause the accuracies of these predictions to break down.

If bacteria evolve resistance to phage attack, this phenotypic change may be costly, such as incurring a reduced growth ability in phage resistant mutants. Such pleiotropic costs of phage resistance should be commonplace, owing to the tendency for phages to evolve specific binding to highly-conserved structures on host cells [27], [28]; altering (or deleting) these binding targets should therefore debilitate bacterial growth. By this logic, simultaneous evolution of cross-resistance to phages that bind to different cellular targets may be more costly for host bacteria, due to evolutionary constraints (i.e., cross-resistance should be unlikely when phages bind differently) combined with the expectation that distinct resistance mutations for each phage will cause fitness to decrease additively or negatively synergistically. Clearly, the evolution of high-cost resistance in target bacteria would be advantageous for phage therapy applications, because this outcome should improve the goal of reducing bacterial load. However, there is mixed evidence for the prediction that simultaneous evolution of resistance to multi-phage cocktails should be more costly for bacteria, relative to fitness costs associated with sequential phage application. Wright et al. [25] observed that sequential resistance was more costly than simultaneous resistance in half of their replicates. Similarly, in a separate study involving additional phage pairs and $P$. aeruginosa strain PA01, in vitro data showed no differences between sequential and simultaneous phage exposure, in terms of bacterial clearance or cost of resistance [29] . Again, the equivocal results for phenotypic costs of resistance against sequential versus simultaneous phages in these studies suggest that cocktails are not necessarily the best therapeutic option; the resulting resistant bacteria may be equally debilitated in growth, whereas only sequentially-administered phages tend to cause predictable underlying genetic changes in resistant mutants [25].

Although phage cocktails are popularly touted in the literature, it is known that the temporal dynamics of multi-phage therapy can be complicated [30]. As an example, phages may be mixed together in equimolar 
amounts and applied simultaneously, but (as with any biological entities) there is no guarantee that the phages will be equivalent in their rates of cell entry, intracellular rates of genome replication and packaging, lysis timings, and burst sizes. These different characteristics would easily cause the phage cocktail to deviate from the intended equal representation of virus types in the mixture as phage particles interact with host cells during therapy, resulting in different relative killing abilities and selection pressures exerted on the bacteria. In theory, any concerns over this possible imbalance could be eliminated if the phages in the cocktail were chosen specifically because they were equal in the various phage traits. However, this notion seems unrealistic, given that even single mutations can radically alter traits of phages that are otherwise isogenic [31], [32]. Moreover, this strategy would likely undermine the generally popular goal of discovering and combining functionally distinct phages into a single cocktail based solely on their different host range (e.g., cell-binding) properties. Aside from differing growth capabilities of phages that can alter the intended equal representation of phages in an administered cocktail, effects of phage-phage intracellular competition can complicate predictions of phage-therapy efficacy. Multiplicity of infection (MOI) is defined as the ratio of phage particles to susceptible bacterial cells, when these microbes are combined. MOI is easily controlled in laboratory experiments with phage and bacteria, because it is trivial to accurately measure particle titers and cell densities to achieve the intended initial ratios [33]. However, MOI cannot be controlled in vivo ; phage titer is known upon delivery, but the exact size of the infecting bacterial population is very difficult to ascertain. This becomes important because elevated MOIs (i.e., ratios exceeding 1.0) allow higher probabilities that multiple phage particles co-infect the same host cell, producing phage-phage competition that can select for virus traits which foster antagonistic interactions between viruses [34]-[37]. An extreme example is the evolution of "cheating" where phages are selected to selfishly utilize proteins coded by other coinfecting viruses, causing both wildtype and non-cheater variants of viruses to be competitively disadvantaged for intracellular growth [38], [39]. The net result is for cheater variants to take over the phage population, which surprisingly can occur even if there is a high phenotypic cost of cheating in the phage. For the latter reason, phage-phage competition can both reduce variability of the overall phage community, as well as decrease the efficacy of phage particle production if phage competitiveness does not correlate with an advantage in burst size [36]. Obviously, the concern over adverse phage-phage interactions become minimized if phages are used alone or sequentially, relative to the complexities that might occur when cocktails are employed. Nevertheless, we note that these and other possible negative consequences of phagephage competition remain poorly studied in phage therapy, making it currently difficult to predict how differing treatment strategies would be affected by the phenomenon.

As planned trials are initiated and completed, the outcomes should allow us to determine the impact of phage therapy on pulmonary infections associated with CF. Individual cases treated by our group and others have suggested a clinical benefit from the use of phage therapy in complicated cases, but until we have data from a controlled clinical trial, we can only speculate.

\section{References}

[1] L. L. Maragakis, E. N. Perencevich, and S. E. Cosgrove, "Clinical and economic burden of antimicrobial resistance.," Expert Rev. Anti. Infect. Ther., vol. 6, no. 5, pp. 751-63, Oct. 2008.

[2] M. E. A. De Kraker, M. Wolkewitz, P. G. Davey, and H. Grundmann, "Clinical impact of antimicrobial

resistance in European hospitals: Excess mortality and length of hospital stay related to methicillin-resistant Staphylococcus aureus bloodstream infections," Antimicrob. Agents Chemother. , vol. 55, pp. 1598-1605, 2011.

[3] M. E. A. de Kraker, P. G. Davey, H. Grundmann, and BURDEN study group, "Mortality and hospital stay associated with resistant Staphylococcus aureus and Escherichia coli bacteremia: estimating the burden of antibiotic resistance in Europe.," PLoS Med. , vol. 8, no. 10, p. e1001104, Oct. 2011.

[4] S. E. Cosgrove, "The relationship between antimicrobial resistance and patient outcomes: mortality, length of hospital stay, and health care costs.," Clin. Infect. Dis. , vol. 42 Suppl 2, pp. S82-9, Jan. 2006.

[5] K. Rowland, "Totally drug-resistant TB emerges in India," Nature, Jan. 2012. 
[6] M. E. Falagas and D. E. Karageorgopoulos, "Pandrug resistance (PDR), extensive drug resistance (XDR), and multidrug resistance (MDR) among Gram-negative bacilli: need for international harmonization in terminology.," Clin. Infect. Dis., vol. 46, no. 7, pp. 1121-2; author reply 1122, Apr. 2008.

[7] U. Theuretzbacher et al. , "Analysis of the clinical antibacterial and antituberculosis pipeline.," Lancet. Infect. Dis. , vol. 19, no. 2, pp. e40-e50, 2019.

[8] D. S. Armstrong et al. , "Lower airway inflammation in infants and young children with cystic fibrosis.," Am. J. Respir. Crit. Care Med., vol. 156, no. 4 Pt 1, pp. 1197-204, Oct. 1997.

[9] E. C. Dasenbrook, C. A. Merlo, M. Diener-West, N. Lechtzin, and M. P. Boyle, "Persistent methicillinresistant Staphylococcus aureus and rate of FEV1 decline in cystic fibrosis.," Am. J. Respir. Crit. Care Med. , vol. 178, no. 8, pp. 814-21, Oct. 2008.

[10] D. Dolce et al. , "Methicillin-resistant Staphylococcus aureus eradication in cystic fibrosis patients: A randomized multicenter study," PLoS One, vol. 14, no. 3, p. e0213497, Mar. 2019.

[11] C. Cigana et al. , "Staphylococcus aureus Impacts Pseudomonas aeruginosa Chronic Respiratory Disease in Murine Models.," J. Infect. Dis. , vol. 217, no. 6, pp. 933-942, 2018.

[12] S. K. Remold, C. K. Brown, J. E. Farris, T. C. Hundley, J. A. Perpich, and M. E. Purdy, "Differential habitat use and niche partitioning by Pseudomonas species in human homes.," Microb. Ecol. , vol. 62, no. 3, pp. 505-17, Oct. 2011.

[13] J. Emerson, M. Rosenfeld, S. McNamara, B. Ramsey, and R. L. Gibson, "Pseudomonas aeruginosa and other predictors of mortality and morbidity in young children with cystic fibrosis.," Pediatr. Pulmonol. , vol. 34, no. 2, pp. 91-100, Aug. 2002.

[14] G. M. Nixon et al. , "Clinical outcome after early Pseudomonas aeruginosa infection in cystic fibrosis.," J. Pediatr., vol. 138, no. 5, pp. 699-704, May 2001.

[15] N. Volkova et al. , "Disease progression in patients with cystic fibrosis treated with ivacaftor: Data from national US and UK registries.," J. Cyst. Fibros. , vol. 19, no. 1, pp. 68-79, Jan. 2020.

[16] K. B. Hisert et al. , "Restoring Cystic Fibrosis Transmembrane Conductance Regulator Function Reduces Airway Bacteria and Inflammation in People with Cystic Fibrosis and Chronic Lung Infections.," Am. J. Respir. Crit. Care Med., vol. 195, no. 12, pp. 1617-1628, 2017.

[17] S. A. Sarker et al. , "Oral T4-like phage cocktail application to healthy adult volunteers from Bangladesh," Virology , vol. 434, no. 2, pp. 222-232, Dec. 2012.

[18] A. Bruttin and H. Brussow, "Human Volunteers Receiving Escherichia coli Phage T4 Orally: a Safety Test of Phage Therapy," Antimicrob. Agents Chemother. , vol. 49, no. 7, pp. 2874-2878, Jul. 2005.

[19] S. McCallin et al. , "Safety analysis of a Russian phage cocktail: from metagenomic analysis to oral application in healthy human subjects.," Virology, vol. 443, no. 2, pp. 187-96, Sep. 2013.

[20] D. D. Rhoads, R. D. Wolcott, M. A. Kuskowski, B. M. Wolcott, L. S. Ward, and A. Sulakvelidze, "Bacteriophage therapy of venous leg ulcers in humans: results of a phase I safety trial.," J. Wound Care , vol. 18, no. 6, pp. 237-8, 240-3, Jun. 2009.

[21] M. Kutateladze and R. Adamia, "Phage therapy experience at the Eliava Institute," Médecine Mal. Infect. , vol. 38, no. 8, pp. 426-430, Aug. 2008.

[22] L. Kvachadze et al. , "Evaluation of lytic activity of staphylococcal bacteriophage Sb-1 against freshly isolated clinical pathogens.," Microb. Biotechnol., vol. 4, no. 5, pp. 643-50, Sep. 2011.

[23] N. Law et al. , "Successful adjunctive use of bacteriophage therapy for treatment of multidrug-resistant Pseudomonas aeruginosa infection in a cystic fibrosis patient.," Infection, vol. 47, no. 4, pp. 665-668, Aug. 2019. 
[24] R. M. Dedrick et al. , "Engineered bacteriophages for treatment of a patient with a disseminated drugresistant Mycobacterium abscessus," Nat. Med., vol. 25, no. 5, pp. 730-733, May 2019.

[25] R. C. T. Wright, V.-P. Friman, M. C. M. Smith, and M. A. Brockhurst, "Resistance Evolution against Phage Combinations Depends on the Timing and Order of Exposure," MBio , vol. 10, no. 5, Sep. 2019.

[26] R. Kassen, "The experimental evolution of specialists, generalists, and the maintenance of diversity," $J$. Evol. Biol. , vol. 15, no. 2, pp. 173-190, Mar. 2002.

[27] D. L. Smith et al. , "Short-tailed stx phages exploit the conserved YaeT protein to disseminate Shiga toxin genes among enterobacteria.," J. Bacteriol. , vol. 189, no. 20, pp. 7223-33, Oct. 2007.

[28] P. E. Kortright, Kaitlyn E., Chan, Benjamin K., Turner, "High-throughput discovery of phage receptors using transposon insertion sequencing of bacteria.," Proc. Natl. Acad. Sci., vol. in press, 2020.

[29] A. R. Hall, D. De Vos, V.-P. Friman, J.-P. Pirnay, and A. Buckling, "Effects of Sequential and Simultaneous Applications of Bacteriophages on Populations of Pseudomonas aeruginosa In Vitro and in Wax Moth Larvae," Appl. Environ. Microbiol. , vol. 78, no. 16, pp. 5646-5652, Aug. 2012.

[30] B. R. Levin and J. J. Bull, "Population and evolutionary dynamics of phage therapy.," Nat. Rev. Microbiol. , vol. 2, no. 2, pp. 166-73, Feb. 2004.

[31] S. Duffy, C. L. Burch, and P. E. Turner, "Evolution of host specificity drives reproductive isolation among RNA viruses.," Evolution, vol. 61, no. 11, pp. 2614-22, Nov. 2007.

[32] M. Dessau, D. Goldhill, R. C. McBride, R. L. McBride, P. E. Turner, and Y. Modis, "Selective pressure causes an RNA virus to trade reproductive fitness for increased structural and thermal stability of a viral enzyme.," PLoS Genet. , vol. 8, no. 11, p. e1003102, 2012.

[33] A. M. Kropinski, A. Mazzocco, T. E. Waddell, E. Lingohr, and R. P. Johnson, "Enumeration of bacteriophages by double agar overlay plaque assay.," Methods Mol. Biol., vol. 501, pp. 69-76, 2009.

[34] P. E. Turner and L. Chao, "Sex and the evolution of intrahost competition in RNA virus phi6," Genetics , vol. 150, pp. 523-532, 1998.

[35] P. E. Turner, "Parasitism Between Co-Infecting Bacteriophages," 2005, pp. 309-332.

[36] J. J. Dennehy and P. E. Turner, "Reduced fecundity is the cost of cheating in RNA virus phi6.," Proceedings. Biol. Sci., vol. 271, no. 1554, pp. 2275-82, Nov. 2004.

[37] R. Froissart, C. O. Wilke, R. Montville, S. K. Remold, L. Chao, and P. E. Turner, "Co-infection Weakens Selection Against Epistatic Mutations in RNA Viruses," Genetics, vol. 168, no. 1, pp. 9-19, Sep. 2004.

[38] S. L. Diaz-Munoz, R. Sanjuan, and S. West, "Sociovirology: Conflict, Cooperation, and Communication among Viruses.," Cell Host Microbe , vol. 22, no. 4, pp. 437-441, Oct. 2017.

[39] P. E. Turner and L. Chao, "Sex and the evolution of intrahost competition in RNA virus phi6.," Genetics , vol. 150, no. 2, pp. 523-32, Oct. 1998. 